\title{
Redesigning an intensive insulin service for patients with type I diabetes: a patient consultation exercise
}

This article was published in the following Dove Press journal:

Patient Preference and Adherence

4 June 2013

Number of times this article has been viewed

\author{
Seyda Ozcan ${ }^{1-3}$ \\ Helen Rogers ${ }^{3}$ \\ Pratik Choudhary ${ }^{3}$ \\ Stephanie A Amiel ${ }^{3}$ \\ Alison Cox $^{3}$ \\ Angus Forbes ${ }^{2,3}$ \\ 'Department of Medical Nursing, \\ Florence Nightingale Nursing \\ Faculty, Istanbul University, Istanbul, \\ Turkey; ${ }^{2}$ Department of Primary \\ and Intermediate Care, Florence \\ Nightingale School of Nursing \\ and Midwifery, King's College \\ London, London, United Kingdom; \\ ${ }^{3}$ Department of Diabetes, King's \\ College Hospital NHS Foundation \\ Trust, London, United Kingdom
}

Context: Providing effective support for patients in using insulin effectively is essential for good diabetes care. For that support to be effective it must reflect and attend to the needs of patients.

Purpose: To explore the perspectives of adult type 1 diabetes patients on their current diabetes care in order to generate ideas for creating a new patient centered intensive insulin clinic.

Methods: A multi-method approach was used, comprising: an observational exercise of current clinical care; three focus groups $(n=17)$; and a survey of service users $(n=419)$ to test the ideas generated from the observational exercise and focus groups (rating 1 to 5 in terms of importance). The ideas generated by the multi-method approach were organized thematically and mapped onto the Chronic Care Model (CCM).

Results: The themes and preferences for service redesign in relation to CCM components were: health care organization, there was an interest in having enhanced systems for sharing clinical information; self-management support, patients would like more flexible and easy to access resources and more help with diabetes technology and psychosocial support; delivery system design and clinical information systems, the need for greater integration of care and better use of clinic time; productive relationships, participants would like more continuity; access to health professionals, patient involvement and care planning. The findings from the patient survey indicate high preferences for most of the areas for service enhancement identified in the focus groups and observational exercise. Clinical feedback and professional continuity (median $=5$, interquartile range $=1$ ) were the most highly rated.

Conclusion: The patient consultation process had generated important ideas on how the clinical team and service can improve the care provided. Key areas for service development were: a stronger emphasis of collaborative care planning; improved patient choice in the use of health technology; more resources for self-management support; and a more explicit format for the process of care in the clinic.

Keywords: service development, type 1 diabetes, patient feedback, quality improvement, intensive insulin treatment

\section{Introduction}

Intensive insulin therapy aims to reduce blood glucose to near normal levels without increasing the risk of excess hypoglycemia or weight gain. ${ }^{1}$ Recent developments in insulin delivery technology, blood glucose monitoring, and patient education have expanded the possibilities for intensifying insulin therapy safely in patients with type 1 diabetes. ${ }^{2}$ However, managing these technologies within the care system to ensure they are used to optimal effect can be challenging. ${ }^{2}$ There are many patients who do not access these programs in a timely way and patients can also find it hard
Correspondence: Seyda Ozcan Department of Medical Nursing, Florence Nightingale Nursing Faculty, Istanbul University,

Abide-i Hurriyet Cad. Sisli,

3438I, Istanbul, Turkey

Tel +90532 I64 6717

Email seyda_ozcan@hotmail.com 
to sustain the benefits of these programs in the longer term. ${ }^{3}$ Therefore, using these technologies effectively requires a package of intervention based on a partnership with the patient and ongoing self-management support. ${ }^{1}$ As the numbers of patients with Type 1 diabetes increases and greater advances in technology are achieved, there will be even more pressure to design services that are responsive to patient needs and are efficient in the use of limited health care resources.

In redesigning a service there are a number of important considerations. There needs to be a theoretical basis to the redesign, an assessment of current provision, and engagement of stakeholders. ${ }^{2-6}$ An important part of this process is to involve patients to examine their current experiences of care and how they would like to see the service develop. This paper reports a novel patient consultation undertaken as part of a service redesign of a tertiary intensive insulin service.

\section{Methods}

The service redesign was comprised of two integrated elements to address the experiences and needs of the patients: firstly, focus group discussions; and secondly, a patient survey. This paper reports the findings of the patient consultation.

\section{Theoretical model}

Service redesign needs to be informed by an underpinning theoretical model to ensure that all the different components of the care system are addressed..$^{5-7}$ The model adopted for this project was the Chronic Care Model (CCM). The CCM is a whole system approach comprised of three key elements: the health care system; community resources; and the development of a productive relationship between a prepared patient and clinician. ${ }^{7-8}$ Within the health care system element there is a need to consider: self-management support; the care delivery design; the clinical information systems; and the clinical decision support systems. ${ }^{9}$ These are areas of high relevance to intensive insulin treatment as self-management, supportive information, and clinical decision making are key elements in the process. While the CCM has been extensively evaluated in diabetes care, the studies have tended to be in type 2 patients based in primary care..$^{4,710}$ Indeed, there are very few studies of care system design in type 1 diabetes and they focus on specific elements of the care system rather than the whole system of care. ${ }^{3,6,11}$ In some ways, insulin intensification is different from other aspects of chronic care as there is a period of high intervention (intensifying) and then maintenance. ${ }^{2}$ Nevertheless, the CCM was adopted as giving a useful reference for examining the components of the insulin service currently provided by the clinic. To our knowledge this is the first time such an approach has been adopted for developing the care provided to patients with type 1 diabetes.

\section{Care setting}

The focus of the re-design was the intensive insulin therapy clinic within a large tertiary diabetes service treating over 1000 people with type 1 diabetes. The intensive insulin clinic had evolved over the years and was largely focused on delivering technology enhanced care and structured patient education. The characteristics of the current clinic are detailed in Figure 1 in relation to the components of the CCM. ${ }^{9}$

In the current model, the multidisciplinary team (three diabetes consultants; two diabetes specialist nurses; two specialist dietitians; and input as required from psychology and psychiatry) provided specialist support for patients to improve their glycemic control and minimize hypoglycemia. This was achieved via: structured education programs (Dose Adjustment for Normal Eating [DAFNE]); top up educational sessions (carbohydrate counting and dose adjustments); a continuous subcutaneous insulin infusion (CSII) service; and the use of blood glucose sensing and monitoring technologies. While this model had been very effective, increasing numbers of patients and greater demands for technology support meant that the care system was struggling to manage demand. The clinical team also recognized that there was scope to improve the efficiency of the service as not all the patients being seen in the intensive clinic were requiring intensification of their glycemic control, either because the intensification had been unsuccessful or because they had achieved their clinical targets. In addition, it was recognized

\begin{tabular}{|ll|}
\hline $\begin{array}{l}\text { Self-management } \\
\text { support }\end{array}$ & $\begin{array}{l}\text { Education programs. } \\
\text { Tele-support from diabetes nurses. } \\
\text { Psychosocial interventions: psychology } \\
\text { and psychiatry with targeted behavior } \\
\text { change interventions. }\end{array}$ \\
Care delivery & Multidisciplinary input (diabetologist, \\
design & diabetes nurse specialists, and dietitians). \\
Clinical information & An electronic patient record with limited \\
systems & interrogable functionality \\
Clinical decision & Technology for assessing blood glucose \\
support systems. & performance. \\
& Multidisciplinary review. \\
Patient/clinician & Patient given care objectives by clinical \\
interface & team documented in a letter copied to the \\
& patient. \\
& Some more in-depth case management \\
& for pump patients. \\
\hline
\end{tabular}

Figure I Key components of current system. 
that there was no formal model for joint care planning with patients. ${ }^{?}$

\section{Aim and questions}

The aim of the patient consultation exercise was to explore patients' perspectives on their current diabetes care and their ideas for improving the service to inform service development. The exercise aimed to address the following questions:

1. How do patients view the current care they receive?

2. What do patients think would improve the care they receive?

A multi-method approach was used for the consultation exercise, comprising: focus groups; a patient survey; and an audit of clinical records. The CCM was used to inform the areas for exploration. ${ }^{9}$

\section{Data collection and participants}

Data were collected in 2011 in two stages.

\section{Stage I: focus groups}

Focus groups were convened to allow a more expansive discussion of the diabetes service. Patients were invited to the groups during consecutive clinic visits $(n=43) ; 17$ patients confirmed to attend and were allocated to one of three groups: multiple daily injection (MDI) therapy; CSII therapy; and a mixed CSII and MDI group. These groups were semistructured and addressed the following topics: experience of clinic visits; interaction with health professionals; selfmanagement support; and their overall perspective on the service and how it could be enhanced. Participants were encouraged to discuss each other's experiences and ideas. The group was led by a facilitator (AF) who, while part of the clinical team, had no prior clinical connection with any of the participants. An observer (SO) was also present, keeping a written record of the group's ideas. Flip-charts were used to generate consensusbased lists of key areas for service development. The focus groups were also tape-recorded and transcribed verbatim.

\section{Stage 2: patient survey}

The survey was constructed with reference to the output of the focus groups and following an observational exercise of current services and discussion with team members. The survey contained questions relating to: demographic characteristics; current diabetes treatment; blood glucose measurement, control and targets; care experience and satisfaction; and what patients wanted to improve in the service. The target participants for the consolation were patients with type 1 diabetes who had used the diabetes service within the last 18 months $(n=585)$. This selection meant that the focus was on patients who were actively receiving care from the service, both in the intensive and general diabetes follow-up clinics. The survey questionnaire was sent to all patients. Patient glycated hemoglobin levels $\left(\mathrm{HbA}_{1 \mathrm{c}}\right)$ were identified from the patients' electronic medical records.

\section{Data analysis}

The content of the focus group transcriptions was coded and analyzed thematically to express the range of ideas voiced by the groups. The coding frame was generated iteratively between the facilitator and the observer until all the content had been coded. The survey data contained nominal and ordinal data, together with some text-based open questions. The numerical data were compiled to provide descriptive statistics detailing patient characteristics and service views and preferences. The open question responses were subject to a content analysis to categorize the responses following a process of content analysis previously described by the authors. ${ }^{12}$ These categories were described and presented with their frequency. The CCM was used to synthesize the content fields and thematic categories from the different data sources.

\section{Results}

The findings of the patient consultation are presented below, in relation to the questions addressed by the development exercise.

\section{Patient characteristics}

Seventeen patients participated in the focus groups $(n=11$ CSII and $n=6 \mathrm{MDI})$. There were nine female and eight male participants. The mean age was $48( \pm 11)$ years, mean diabetes duration was $29( \pm 14)$ years, and the median of visits to the service in the last 12 months was two. Four hundred and nineteen patients returned the patient survey. This response represented $71.6 \%$ of the patients who had attended the service in the last 18 months. The mean travelling time to the clinic was $60( \pm 44)$ minutes, with $22 \%$ of patients living more than 90 minutes away. The demographic and clinical characteristics of the patients are summarized in Table 1.

\section{Strengths, weaknesses, and service satisfaction}

The survey asked patients to identify the strengths and weaknesses of the current diabetes service. The themes from their responses are summarized in Table 2 . The greatest strength was the expertise and support of the multidisciplinary team. 
Table I Characteristics of survey respondents

\begin{tabular}{|c|c|c|}
\hline Demographic & & Range \\
\hline & Mean (SD) & \\
\hline Age & $48(15)$ & \\
\hline Gender & n (\%) & \\
\hline Male & $147(36)$ & \\
\hline Female & $266(64)$ & \\
\hline \multicolumn{3}{|l|}{ Ethnicity } \\
\hline White & $367(87)$ & \\
\hline Black & $13(3)$ & \\
\hline Asian & $9(2)$ & \\
\hline Other & $15(8)$ & \\
\hline \multicolumn{3}{|l|}{ Relationship } \\
\hline Single & $103(25)$ & \\
\hline Married & $262(64)$ & \\
\hline Separated & $40(10)$ & \\
\hline \multirow[t]{2}{*}{ Widowed } & $6(1)$ & \\
\hline & Mean (SD) & \\
\hline Diabetes duration (years) & $26(15)$ & $1-72$ \\
\hline Clinical & Mean (SD) & \\
\hline $\mathrm{HbA}_{\mathrm{Ic}}$ & $7.6(1.3)$ & $4-16$ \\
\hline Insulin therapy & n (\%) & \\
\hline MDI & $266(64)$ & \\
\hline CSII & 137 (33) & \\
\hline BD mix & $12(3)$ & \\
\hline Service & Mean (SD) & \\
\hline Duration of time (years) in clinical service & $16(15.4)$ & $0-67$ \\
\hline \multirow[t]{2}{*}{ How many visits in past 12 months } & $3(3.4)$ & $1-30$ \\
\hline & n (\%) & \\
\hline Number of missed appointments & $77(20)$ & \\
\hline
\end{tabular}

Abbreviations: $\mathrm{BD}$ mix, bi-daily mix; CSII, continuous subcutaneous insulin infusion; $\mathrm{MDI}$, multiple daily injections; $\mathrm{SD}$, standard deviation; $\mathrm{HbA}_{\mathrm{Ic}}$, Hemoglobin $\mathrm{A}_{\mathrm{Ic}}$.

Table 2 Strengths and weaknesses of current service

\begin{tabular}{ll}
\hline & $\mathbf{n}(\%)$ \\
\hline Strengths & \\
Expertise and support of MDT & $187(45)$ \\
Patient involvement and & $88(21)$ \\
Quality of clinical service & $27(6)$ \\
Educational support & $19(5)$ \\
Telecare support & $13(3)$ \\
Accessibility and responsiveness & $10(2)$ \\
Care integration & $5(1)$ \\
Unsure & $12(3)$ \\
Missing & $53(14)$ \\
Weaknesses & \\
Clinic organization & $156(37)$ \\
No weaknesses & $40(10)$ \\
Care integration & $38(10)$ \\
Continuity & $37(9)$ \\
Access to MDT & $14(3)$ \\
Holistic model & $13(3)$ \\
Psychological support & $11(3)$ \\
Technology support & $11(3)$ \\
Responsiveness of telecare & $9(2)$ \\
Emergency support & $2(1)$ \\
Other & $12(3)$ \\
Missing & $73(16)$ \\
\hline Abrrevation:MDT, mudscip
\end{tabular}

Abbreviation: MDT, multidisciplinary team.
Patients identified individual team members and felt that the team was good at responding to their needs and was supportive and encouraging. They also valued the technical skills of the team. This theme was followed by patient involvement in care, which encompassed both being included in care planning and personalizing care. Some patients particularly valued the relationship they had established with individual team members. Other areas of strength included the responsiveness of the service (being able to get access to help when they needed it); the telecare support provided (advice through email and telephone); and specific technology support (mainly pump related).

In terms of weaknesses, patients felt that the organization of the clinic was the main area where there were difficulties. These were generally related to clinic waiting times (too long) and appointments (being able to get them when needed). Other areas included: a lack of continuity (seeing the same professional, particularly the same doctor); weaknesses in integration between general practitioner (GP) care and eye care; access to technology like continuous blood glucose monitoring; delays in feedback through telecare; access to the multidisciplinary team; access to psychological care; and emergency support.

Overall patients reported high levels of satisfaction with the clinical service they received with a median rating of 5 (interquartile range $[\mathrm{IQR}]=1)(1=$ not at all satisfied, $5=$ very satisfied). Similar ratings were given for satisfaction with input from the doctors and nurses in the clinical team. A lower level of satisfaction was expressed for the clinic waiting time ( median $=3, \mathrm{IQR}=2$ ).

\section{Service development}

The focus groups and survey data generated a number of themes that expressed the views of patients in relation to the current service and how it could be developed. The themes included: the experience of clinic visits; interaction with health professionals; self-management support; and care integration. The survey data presented here were in response to the open question "what one thing would improve the diabetes service you receive," to which 315 (75\%) patients gave a valid response. The number of survey respondents to each area, together with quotes from the focus groups and survey data, are presented in Table 3 . The table also identifies the linkage between the themes and the areas of CCM.

\section{Clinic visits}

Patients identified a number of different issues relating to clinic visits, focusing on: the preparation for the visit and 
Table 3 Development themes, data extracts, and survey responses $(n=315)$

Theme - CCM area

Clinic visits and organization of clinic - delivery system design

Appointment systems

SD: "more flexible later appointments;" "interactive booking systems;" "producing a leaflet guide

to all the diabetes services available with relevant phone numbers for self-referral;"

"one stop shop for all health appointments could be co-ordinated to save time."

FG: "I get appointment letters, reminder letters, that's good. But they just say 'diabetes.'

And it can be a nurse appointment or a doctor. And I just don't know what they are."

Clinic organization

(maximizing the time)

FG: "So it's something about making sure that all the information that's needed for the consultation is on the table, ready to roll when you're actually going to have the dialogue, rather than, 'Oh we need this, we need that, and, you know, it won't work so well."

FG: "If there were two rooms available, one waiting for people watching the telly if that's what they want to do for their own well-being, others might want to go in an education room, and there could be videos on the telly about products or ways to handle certain things.

But they wouldn't be forced on people, is what l'm saying."

Interaction with health professionals - productive relationships (patient/professional)

Continuity

FG: "I would prefer to see the same doctor each time."

SD: "Seeing the same clinician so they can follow your care plan."

Access to health

professional

FG: "I call the nurses up...very helpful talking to her, because sometimes, you know, when you're really high and you've been doing great for a while, it's hard to think clearly, isn't it."

SD: "More time from professionals to check on me between appointments."

Patient involvement SD: "Being able to talk and ask questions freely without feeling you are taking up too much time;" "Trying to build up a relationship with doctor and nurse;" "Doctor very passionate about his subject but needs to explain in layman's terms;" "Take more time to listen to patients."

FG: "I think what would be really useful is if you had a plan, that each doctor updated a plan that they discussed with you in terms of what you're going to do ... I think that would be really, really useful, because then you could coordinate everything."

Self-management support - self-management support

Technology support

SD: "More available help on using the pump;" "Keeping us up-to-date with latest technology. I would like a way of knowing my sugar levels 24 hours a day (like a watch);" "Updates on the latest treatments."

FG: "Use of and support for technology is so big in this hospital, I think we should have somebody who is on call on clinic day, so that they can talk about some aspects of using electronic products. So it's not just the doctors and nurses."

Telecare use support $\quad$ FG: "For email contact, the response should preferably be the same day, when you need it." SD: "Faster response time to email enquiries and telephone messages, sometimes this takes 48 hours plus."

Educational resources $\quad$ FG: (about DAFNE) "It's brilliant, it is brilliant. It's a little group like this and everybody talks and it really is fantastic."

SD: "It would be good to be part of a support network of diabetics of a similar age so you could discuss and; compare experiences - especially when it comes to exercise;"

"Information of educational classes available - when and where!"

Emergency support $\quad$ FG: "I've rung the GP, I've rung NHS Direct, l've rung all sorts of people but they were not able to help me...having emergency numbers would be good."

SD: "Perhaps a between appointment online trouble shooting service for unpredictable problems." SD: "Better understanding of the emotional impact of diabetes."

Psychosocial support FG: "I think it all comes down to sort of emotional and mental support, which I know that doctors and nurses now are fully aware, you know."

Care integration - delivery system design

Holistic model of care

SD: "Any complication arises like heart problem, kidney, eyes should all be looked after in the same hospital so they can communicate easily with other specialists and would not be extra time consuming like trying to find out other results."

Integration of services FG: "I do wish that all the facilities were here though, so that I didn't have to get a letter from my doctor basically and have to have the same tests again."

Not able to identify anything specific to improve.

These comments were generally saying just keep providing the current service.
Response rate

84 (27)

\section{te}

(1)


organization of appointments; the experience of attending the clinic; and the facilities within the clinic.

Many patients in the survey reported issues relating to appointments, identifying the need for: greater control over appointments; being able to book appointments (including an online booking system); having more frequent appointments tailored to their needs; greater availability of appointments in the evenings and on weekends; and coordinated appointments to avoid multiple attendances. A few patients found the text reminders for their appointment helpful. Patients did not always find the information they were given prior to their visit helpful, as it was not always clear what the visit was for and who they would see. Patients thought it would be better if blood tests and downloaded data from pumps and glucose meters were available prior to the consultation.

Patients can experience long waiting times and this was an area that patients felt could be improved. Patients in the focus groups reported that they were generally prepared for a long wait and thought it was a fair tradeoff for the receipt of a high quality consultation. They also suggested the waiting time could be used more productively by: doing some of the preparation for the consultation (eg, downloading pumps); having a space for social and educational interaction; and having a technology resource where patients could have access to new technologies with support in troubleshooting problems or expanding their use of a technology (eg, learning how to download and read their pump or glucose meter).

\section{Interaction with health professionals}

Interaction with health professionals is core to the patient's care experience and participants identified areas for development in relation to: continuity; access to health professionals; patient involvement; and care planning.

Continuity was expressed in two dimensions: continuity of professional; and continuity of care (information and planning of care). In terms of professionals, patients preferred to see the same health professional where possible. Continuity of care related to the follow through of the patient's care plan. This continuity can break down if there is inadequate follow-up or inconsistencies between professionals in addressing the care plan.

Patients also wanted more interaction with health professionals and greater flexibility in that interaction. It was suggested by one respondent that a drop-in clinic (no appointment required) would be useful. In the survey, patients were asked whether their review with the doctor was adequate (currently it is a minimum annual review): 272 (58\%) felt it was about right; and 199 (42\%) thought it was too little.
Patients valued being involved in their care and desired collaborative interactions with health professionals. In part this was related to good communication (being listened to), but involved care planning and shared decision making. Patients also liked clear feedback from the health professional.

Patients were asked more specific questions in relation to their involvement in their care: $76 \%(n=302)$ had been involved in developing their own care plan; $80 \%(n=345)$ felt they had been involved in their diabetes care as much as they wanted; $54 \%(n=225)$ felt that their care plan was taken into account by health professionals; $61 \%(\mathrm{n}=256)$ felt that the professionals had a good understanding of their needs; and $27 \%(n=115)$ felt they received enough emotional support from the diabetes team.

\section{Self-management support and education}

In terms of self-management support, patients identified a number of important areas that included: technology support; telecare use; educational resources; psychological support; and social support.

Technology support relates to the different needs of patients in using technology to enhance their selfmanagement practices. The range of technology that patients identified included: pumps, continuous glucose monitoring (CGM), and blood glucose monitoring. Patients would like more choice over technology selection and experienced problems in accessing technology. These problems included: inconsistent local advice and procedures (such as the level of expertise in the local area and local rules on funding pumps); and limitations in relation to changing and updating technology. There were also issues of compatibility with the patient's own equipment, such as their computer. Patients would like more support in understanding the functionality of the technology and in interpreting the data generated by their meters and pumps. A few patients also wanted more advanced technologies such as closed loop systems.

Patients in the focus groups had a broad range of views on telecare support. While most saw advantages in communicating through different media (telephone, email, text, and web), they also reported that tele-access to professionals could be frustrating when it was difficult to access the person they needed help from or if the person was unsure or unable to address their problem. Patients expressed concern that services could become too technology-based, as some patients do not use the internet and prefer more traditional modes of interaction. Patients also suggested that telecommunication preferences varied between telephone, texts, 
and emails. Survey respondents also emphasized the need for easy access to help and quick replies to address their needs, whether this is by phone or email. Patients were asked to rate different modes of communication (see Table 4), with face to face communication being most strongly rated and most commonly experienced. Emails were also viewed quite positively, whereas there was more ambivalence toward text and telephone contact. Female respondents gave higher utility ratings to the electronic communication methods compared to males and had higher usage, particularly for text messaging $(P<0.05)$.

Patients found the educational support provided to be very helpful, particularly those who had attended structured programs like DAFNE. However, they also emphasized the need for an ongoing program of education so they can refresh and extend their skills, particularly by learning from each other. Patients would like to have the education program more visibly defined so they can see what is available and also check to see what they have done. They also pointed out that running the program during working hours is difficult for some patients.

Patients commented on a lack of easy access to rapid diabetes support in situations of acute need (eg, intercurrent illness or equipment failure). Most patients had experienced episodes where they needed urgent advice to manage or troubleshoot a diabetes problem. They believed that if they had access to a diabetes support system to address these problems, things would be less likely to go wrong and they would have less need for emergency services. In fact, the service dose provide 24-hour phone support service, but only $91(22 \%)$ respondents had used this service.

\section{Care integration}

Care integration was important to patients, they identified two specific areas: integration between services and integration of their diabetes with their general health care.

Patients identified a number of areas where the interface between services can break down: primary and secondary care; eye screening; pregnancy; and foot care. Patients find it frustrating when their GP repeats tests already done at the hospital and when the results of tests performed by their GP are not available in the diabetes clinic. Patients would prefer that eye screening was part of their diabetes care. They believe this would be easier for them and also better in terms of getting feedback from the screening as part of their diabetes care.

Patients also felt that their care lacked a holistic approach and did not always attend to their wider health care needs, even though many of these were related to their diabetes. Older participants in the focus groups were aware that age related changes were impacting on their diabetes, affecting their response to insulin, exercise, and diet.

\section{Priorities for development}

In the focus groups, the patients made some specific recommendations for improving the service; these included:

- Regular updates on appointment waiting time in clinic.

- Improved continuity with doctor appointments.

- More information about what you can expect to happen and who you will see at each clinic visit.

- More support in understanding the results of pump and meter downloads and CGM.

- A clear written plan of care at the end of each visit.

- Educational support to help patients maximize the potential of their technology.

- A regular review of their technology to ensure it is suited to their needs, with options to change.

- Patient choice over mode of follow-up communications (letter, email, or text).

- A dedicated website with information and facility for interaction with other patients.

- Being able to book a tele-consultation at a designated time with the diabetes nurse to go through things.

These suggestions, together with other ideas expressed in focus group discussions were incorporated into the survey, and patients were asked to rank their importance from 1 (no importance) to 5 (high importance) (see Table 5). Items were also categorized according to CCM components.

Table 4 Rating of different methods of communication

\begin{tabular}{|c|c|c|c|c|c|c|}
\hline & \multicolumn{3}{|c|}{ Rating median (IQR) ${ }^{\mathbf{a}}$} & \multicolumn{3}{|c|}{ Have used n (\%) } \\
\hline & Total & Female & Male & Total & Female & Male \\
\hline Face-to-face & $5(0)$ & $5(0)$ & $5(0.8)$ & $383(93)$ & $246(92)$ & $137(93)$ \\
\hline Telephone & $4(2)$ & $5(I)$ & $4(2)$ & $202(49)$ & $134(50)$ & $68(46)$ \\
\hline Email interaction & $5(1)$ & $5(1)$ & $4(1)$ & $195(47)$ & $134(50)$ & $6 I(4 I) *$ \\
\hline Text messaging & $4(3)$ & $5(2)$ & $3(3)$ & $109(26)$ & $78(29)$ & $3 I(2 I)$ \\
\hline
\end{tabular}

Notes: aPatients were only rated if they had experienced the communication method $(I=$ not useful, $5=$ very useful $)$. $* P<0.05$.

Abbreviation: IQR, interquartile range. 
Table 5 Ranked service development preferences

\begin{tabular}{llll}
\hline Development area & $\mathbf{n}$ & Median & IQR \\
\hline Getting feedback on clinical results & 409 & 5.00 & I \\
Seeing the same professional each & 410 & 5.00 & I \\
visit (continuity) & & & \\
Having blood test done before appointment & 408 & 4.5 & 2 \\
Knowing which professional you will see & 410 & 4.00 & 2 \\
in the clinic & & & \\
A choice of which professional you see & 407 & 4.00 & 2 \\
More support with diabetes technology & 402 & 4.00 & 2 \\
An email or web-based query service & 399 & 4.00 & 2 \\
Text messages to remind appointments & 399 & 4.00 & 3 \\
An interactive help line & 399 & 4.00 & I \\
Out of hours clinic and education & 359 & 4.00 & I \\
Having a written care plan & 401 & 3.00 & I \\
Web resources & 391 & 3.00 & I \\
Online booking for education programs & 396 & 3.00 & 2 \\
Having education in the waiting area & 406 & 3.00 & 2 \\
More psychological support & 399 & 3.00 & 2 \\
Information on welfare benefits & 397 & 3.00 & 3 \\
and social support & 396 & 3.00 & 3 \\
Support with employment issues & & & \\
\hline Abbreviation: & & & \\
\hline
\end{tabular}

Abbreviation: IQR, interquartile range.

All patients wanted good feedback on their clinical performance and improved continuity.

\section{Discussion}

The data generated by this consultation provide some important insights into what patients need and want from their diabetes team in supporting their self-management. To our knowledge, this is the first study that has explored the need of patients with type 1 diabetes using the chronic care model (CCM). While these data were drawn from only one treatment center, the center is one of the largest in the UK and has a wide geographical distribution of patients. Using the CCM as a framework, it is possible to translate these insights into areas for service redesign. ${ }^{9}$ In terms of the health care system, there are number of areas that could be enhanced.

For self-management support, while there are common underpinning needs such as greater access and more frequent self-management support, there are differences in the preferred mode of interface. The challenge for the service redesign will be in trying to accommodate these different needs. While it may be more progressive to develop webbased and telecare support, there seems to be some resistance to this within the patient population. Patients prefer face to face support from professionals and other patients. Perhaps the way forward would be to introduce tele-systems systematically, with more explicit guidelines for patients in terms of how and when to use these tele-support systems. ${ }^{3,13}$
For example, a change made following this project has been to introduce a booking system for telephone appointments so that patients know when to expect a call. Likewise, the increasing complexity of technology available to patients is changing their self-management behaviors and the support they need. The data suggest that some patients may need additional support in optimizing their use of these technologies. The team has now started to run additional courses helping patients use their technology to greater effect. The choice of technology may also be important for patients, ensuring that they are supported in using a technology that is suited to their clinical needs and to their own capacity to use that technology. ${ }^{2,13}$ In terms of downloading pump information, for example, patients may need some help to interpret these outputs or have some explanation of the output to ensure that they get the feedback they need to increase their selfmanagement performance.

In developing the care delivery system, the key message from patients is that they would like more control over the way they use the service. While they are generally very satisfied with the clinical care they get, they would prefer to be able to direct their appointments to meet their specific needs. Currently, organization of care is orientated toward the service rather than the patient. This will change with the general move toward patient involvement in their care planning, giving patients more control over the way they use the clinic resources. ${ }^{7,11,14}$ However, further investment is required in system infrastructure to maximize patient choice. There would also need to be some safety measures to ensure patients did not disengage as they were used to being instructed in what to do, giving the advantage to more proactive patients. Indeed, if the system was geared solely to patient choice this could mean that the system becomes inefficient, with patients booking into resources they did not need. Therefore, the way forward could be to book the clinical engagements for the year as part of the care planning process in negotiation with the professional. This theme of choice and negation for the use of clinical resources emphasizes the need for stronger case management for some patients, particularly those going through a transitional process such as intensification of their insulin. ${ }^{2,11,13}$

An additional area for the service to consider in terms of system design, is how to incorporate the patients' wider health care needs such as in aging, or during adolescence. ${ }^{2,6,11,15}$ While in some ways, a level of clinical segmentation is inevitable in specialized medicine, there are wider factors in the patients' health that are very relevant to their diabetes management. ${ }^{2,4,13}$ Key factors that may need greater emphasis are the way the service 
addresses the effects of aging on the metabolic needs of type 1 patients as the number of older adults with type 1 diabetes increases.

In terms of the clinical information system, the most important area highlighted by the patients was the need for feedback on their clinical results in a way that they can understand and address in their ongoing care. ${ }^{8}$ For some, there is a strong desire for understanding their relative risk and their relative performance in relation to others. However, again caution is required as there is evidence to show that interpretation of risk varies between patients and relative performance; while motivating for some, may be demotivating for others. ${ }^{16}$ This again emphasizes the importance of the care planning process within the system and the need to give patients some control over the types of information they are exposed to. ${ }^{14}$

Clearly the most important part of the care management process for patients is their interaction with the health professional. ${ }^{6,7}$ A specialist intensive insulin service should consist of staff with a special interest in intensive insulin and pump therapy with clinical, psychological, and educational expertise and other elements of care for people with diabetes. The patients in the current service value the skill and expertise of the team, something that has been highlighted in previous studies. ${ }^{2,3,17}$ In terms of areas for development, the most important factor is continuity of care. ${ }^{16}$ In a busy and complex care system, this can be difficult to secure and different members of the team may be more appropriate for different patient needs. As previously identified, developing a case management protocol as part of the care planning process may help enhance this aspect of care delivery. ${ }^{7}$

One part of the CCM model not addressed in the analysis is that of community resources. ${ }^{8}$ While this component of the model is more relevant in primary care settings, there are some aspects that are relevant to specialist insulin care. The patients identified the clinic itself as a potential resource and would like greater opportunities to interact with other patients and to have self-management resources available in the clinic.

There are a number of limitations in relation to a patient consultation approach to service development that need to be considered. Patients may have expectations from their health services that may not always be realistic. This project also highlights that there are variations between patients in terms of what they want and value. Accommodating the views of all can be problematic as they are not mutually compatible, with patients having varying needs and responding differently to different modes of interaction and clinical resources. In terms of limited health care resources, it is also important to consider the clinical needs of the individual patient. Hence, in developing the service, consideration has also been given to the clinical profile of the patients in the service. The clinical data show that there are patients who require different levels of management and support in achieving and then maintaining optimal glycemic control and management of hypoglycemia. Therefore, in redesigning the service, consideration is also being given to modeling patient activity in the service to enable more clinical resources to be targeted at patients during the intensification process. In recognition of this issue, the team is supporting patients in developing their self-management skills so that on completion of the intensive phase of their treatment, they are well equipped to maintain their control. ${ }^{1,2}$ The team is expanding the self-management resources available to patients and is also developing web-based resources.

\section{Conclusion}

This qualitative study has generated many ideas from patients as to how they can be better supported in managing their diabetes. By using the CCM as framework for the analysis, it has been possible to organize these ideas into strategic areas to inform the service development plan. This development plan includes: the introduction of more collaborative care planning; improved patient choice in the use of health technology; more resources for self-management support; and providing patients with more details on how the clinic works, what is available, and what they can expect from the clinic. The analysis has also highlighted challenges in meeting the diverse needs of patients and how patient choice needs to be considered with the clinical requirements of the patient and service as a whole.

\section{Acknowledgments}

This project was supported with the FEND Fellowship by the Foundation of European Nurses in Diabetes and funded by Beta Cell Trust. The project data was presented in the Diabetes UK Meeting that was held on March 7-9, 2012 in Glasgow, Scotland. ${ }^{18}$

\section{Disclosure}

The authors report no conflicts of interest in this work.

\section{References}

1. National Institute for Clinical Excellence [webpage on the Internet] NICE Clinical Guidelines - CG15: Type 1 diabetes: Diagnosis and management of type 1 diabetes in children, young people and adults London: National Institute for Clinical Excellence; 2004. Available from: http://www.nice.org.uk/CG15. Accessed January 3, 2013.

2. Goenka N, Turner B, Vora J; Diabetes UK Task and Finish group. Commissioning specialist diabetes services for adults with diabetes: summary of a Diabetes UK Task and Finish group report. Diabet Med 2011;28(12):1494-1500. 
3. Renders CM, Valk GD, Griffin SJ, Wagner EH, Eijk JT, Assendelft WJ. Interventions to improve the management of diabetes mellitus in primary care, outpatient and community settings. Cochrane Database Syst Rev. 2001;1:CD001481.

4. Si D, Bailie R, Connors C, et al. Assessing health centre systems for guiding improvement in diabetes care. BMC Health Serv Res. 2005;5:56

5. Collins MM, O'Sullivan T, Harkins V, Perry IJ. Quality of life and quality of care in patients with diabetes experiencing different models of care. Diabetes Care. 2009;32(4):603-605.

6. Dovey-Pearce G, Hurrell R, May C, Walker C, Doherty Y. Young adults' (16-25 years) suggestions for providing developmentally appropriate diabetes services: a qualitative study. Health Soc Care Community. 2005;13(5):409-419.

7. Borgermans L, Goderis G, Ouwens M, Wens J, Heyrman J, Grol RP. Diversity in diabetes care programmes and views on high quality diabetes care: are we in need of a standardized framework? Int J Integr Care. 2008;8:e07.

8. Bodenheimer T. Interventions to improve chronic illness care: evaluating their effectiveness. Dis Manag. 2003;6(2):63-71.

9. Wagner EH. Chronic disease management: what will it take to improve care for chronic illness? Eff Clin Pract. 1998;1(1):2-4.

10. Musacchio N, Scher AL, Giancaterini G, et al. Impact of a chronic care model based on patient empowerment on the management of Type 2 diabetes: effects of the SINERGIA programme. Diabet Med. 2011;28(6):724-730.
11. Snow R, Fulop N. Understanding issues associated with attending a young adult diabetes clinic: a case study. Diabet Med. 2012;29(2):257-259.

12. Forbes A, While A, Taylor M. What people with multiple sclerosis perceive to be important in meeting their current needs. J Adv Nurs. 2007;58(1):11-22.

13. Rasmussen B, Ward G, Jenkins A, King SJ, Dunning T. Young adults' management of Type 1 diabetes during life transitions. J Clin Nurs. 2011;20(13-14):1981-1992.

14. Department of Health. Care Planning in Diabetes: Report from the Joint Department of Health and Diabetes UK Care Planning Working Group. London: Department of Health; 2006. Available from: http:// www.diabetes.org.uk/Documents/Reports/CarePlanningDec06.pdf. Accessed January 27, 2013.

15. Smith SM, Allwright S, O’Dowd T. Effectiveness of shared care across the interface between primary and specialty care in chronic disease management. Cochrane Database Syst Rev. 2007;3:CD004910.

16. Nair KM, Dolovich LR, Ciliska DK, Lee HN. The perception of continuity of care from the perspective of patients with diabetes. Fam Med. 2005;37(2):118-124.

17. Forbes A, While A. The nursing contribution to chronic disease management: A discussion paper. Int J Nurs Stud. 2009;46(1): 119-130.

18. Ozcan S, Forbes A, Rogers H, Choudhary P, Amiel SA, Cox A. Redesigning an intensive insulin service for patients with Type 1 diabetes: a patient engagement exercise. Diabetic Medicine. 2012; 29(Supp1 1): p 144.
Patient Preference and Adherence

\section{Publish your work in this journal}

Patient Preference and Adherence is an international, peer-reviewed, open access journal focusing on the growing importance of patient preference and adherence throughout the therapeutic continuum. Patient satisfaction, acceptability, quality of life, compliance, persistence and their role in developing new therapeutic modalities and compounds to

\section{Dovepress}

optimize clinical outcomes for existing disease states are major areas of interest. This journal has been accepted for indexing on PubMed Central. The manuscript management system is completely online and includes a very quick and fair peer-review system. Visit http://www.dovepress.com/ testimonials.php to read real quotes from published authors. 